\title{
CXCR7 expression in esophageal cancer
}

\author{
Michael Tachezy ${ }^{1 *}{ }^{*}$, Hilke Zander ${ }^{1 \dagger}$, Florian Gebauer ${ }^{1}$, Katharina von Loga ${ }^{2}$, Klaus Pantel ${ }^{3}$, Jakob R Izbicki ${ }^{1}$ \\ and Maximilian Bockhorn ${ }^{1}$
}

\begin{abstract}
Background: The chemokine CXCL12 and its receptor CXCR4 play a major role in tumor invasion, proliferation and metastasis in different malignant diseases, including esophageal carcinoma, amongst others. CXCR7 was recently identified as a novel alternate receptor for CXCL12. The aim of this study was to evaluate the prognostic impact of expression of chemokine receptor CXCR7 in patients with esophageal carcinoma (EC).

Methods: Expression of CXCR7 in primary tumors, lymph nodes and distant metastases of 299 patients with EC was evaluated by immunohistochemistry on a tissue microarray and compared with clinical and histopathological data.

Results: In esophageal cancer sections, CXCR7-specific reactivity was apparent in 45\% of the squamous cell carcinomas (ESCC), but only occasionally in adenocarcinomas. No correlation between CXCR4 and CXCR7 expression was evident. We correlated expression with clinical and histopathological characteristics, but could not find any association.

Conclusions: Contrary to the other known CXCL12 receptor, CXCR4, CXCR7 is expressed in ESCC only, underlining the divergent mechanisms and backgrounds of EAC and ESCC. The results of the study do not indicate a significant functional role for CXCR7 in EAC or ESCC of the esophagus. However, its variable expression in the main two main types of EC needs to be further investigated.
\end{abstract}

Keywords: CXCR4, CXCR7, CXCL12, Esophagus, Esophageal cancer

\section{Background}

Esophageal carcinoma (EC) is one of the most aggressive solid tumors and has an enormous malignant potential for local invasion and early dissemination, resulting in a high rate of tumor recurrence after surgical treatment that is intended to be curative [1-3]. Any effects of multimodal therapeutic approaches such as neoadjuvant radiochemotherapy on survival and quality of life seem to be modest and are therefore not generally accepted $[4,5]$. Surgery remains the treatment of choice for resectable EC $[6,7]$. Biologic, targeted therapies have not found their way into the clinical routine yet, although some promising targets have been identified [8].

Previous reports have shown a crucial role of chemokines and their receptors in tumor growth, angiogenesis, and tumor cell homing in lymph nodes, bone marrow and distant metastases [9-11]. In particular, the chemokine CXCL12 (SDF-1) is a broadly expressed cytokine that

\footnotetext{
* Correspondence: m.tachezy@uke.de

${ }^{\dagger}$ Equal contributors

${ }^{1}$ Department of General, Visceral and Thoracic Surgery, University Medical Center Hamburg-Eppendorf, Martinistrasse 52, 20246 Hamburg, Germany Full list of author information is available at the end of the article
}

plays important roles in embryogenesis, hematopoiesis and tumorigenesis of various entities [12,13]. At present, two receptors of SDF-1 $\alpha$ have been identified, CXCR4 and CXCR7, which may act as contributing factors in metastasic spread of melanoma, breast, gastric, and EC $[12,14]$. While several authors have investigated CXCR4 expression, its function and prognostic impact in EC, no data are available about the expression of CXCR7 in EC [3,15-20]. Inhibition of the CXCR4 receptor by specific antagonists has shown an anti-proliferative effect in EC and other entities in vitro and in vivo in xenograft mouse models $[13,15,16,21]$. Phase 1 trials are investigating the inhibitory potential of CXCR4 antagonists in vivo in several other entities (for example high-grade glioma; NCT01339039 or multiple myeloma; NCT00903968).

Since CXCR7 is the other known receptor for SDF-1 $\alpha$, the question must be raised whether CXCR7 has a similar function as CXCR4 in the tumor cell biology of EC. Therefore, the aim of this study was to investigate the expression of CXCR7 in a large number of EC specimens and to evaluate its potential role as a prognostic and therapeutic target molecule.

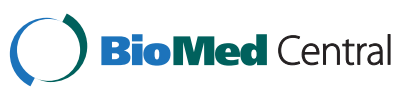

(c) 2013 Tachezy et al.; licensee BioMed Central Ltd. This is an Open Access article distributed under the terms of the Creative Commons Attribution License (http://creativecommons.org/licenses/by/2.0), which permits unrestricted use, distribution, and reproduction in any medium, provided the original work is properly cited. 


\section{Patients and methods}

\section{Study design and patients}

For this study, 299 patients with EC who underwent surgery at University Medical Center Hamburg-Eppendorf between February 1992 and May 2005 were chosen according to available tumor material. None of the patients received neoadjuvant or adjuvant treatment. All data including sex, histology, depth of tumor invasion, lymph node metastasis, tumor type, disease stage (UICC 7th edition) and routinely performed screening for DTC in bone marrow were obtained from a combination of clinical and pathological record review, outpatient clinic medical records and communication with patients and their attending physicians [22].

This study was approved by the ethics committee of the chamber of physicians, Hamburg, Germany. Written informed consent was obtained from all patients to use their resected tumor.

\section{Tissue microarray (TMA)}

The pre-existing tissue microarray consisted of 299 primary lesions and corresponding lymph nodes $(n=147)$ and distant metastases $(n=46)$ from patients with $E C$, together with 10 specimens of healthy esophagus mucosa $[23,24]$. After surgical resection, the specimens were fixed in $4 \%$ buffered formalin, paraffin embedded, and used for TMA construction as previously described [23]. Briefly, hematoxylin-eosin stained sections were made from selected primary tumor blocks (donor blocks) to define representative tumor regions. Tissue cylinders $(0.6 \mathrm{~mm}$ in diameter) were then punched from that region of the donor block using a homemade semi-automated tissue arrayer. Three-micron sections were cut using the Paraffin Sectioning Aid System (Instrumentics, USA).

\section{Immunohistochemistry and scoring}

The CXCR7 staining protocol was optimized in an extensive and standardized multi-step procedure on various benign and malignant tissues; the protocol was modified until selective staining with the lowest background signals was established [25]. Freshly cut TMA sections were analyzed on one day in a single experiment. After deparaffination and drying overnight at $37^{\circ} \mathrm{C}$, sections were rehydrated in Tris-buffered saline $(\mathrm{TBS}=0.05 \mathrm{M}$ Tris- $\mathrm{HCl}$ at $\mathrm{pH} 7.6$ and $0.15 \mathrm{M} \mathrm{NaCl}$ ). CXCR7 pretreatment was performed with target retrieval solution (Dako) at $121^{\circ} \mathrm{C}$ for 5 minutes. Sections were blocked with human AB serum (Biotest Diagnostics, Dreirach, Germany) diluted 1:10 in TBS for 20 minutes. Primary CXCR7 antibody was used at a 1:150 dilution (IgG2a, Clone $358426, \mathrm{R} \& D$ Systems) overnight at $4^{\circ} \mathrm{C}$. A standard indirect immunoperoxidase procedure was used for visualization of bound antibody (Envision System, DAKO). Diaminobenzidine was used as the chromogen. Sections were counterstained with hematoxylin solution (Merck, Darmstadt, Germany).

Complete TMA sections were scanned with a Zeiss MIRAX (Carl Zeiss MicroImaging GmbH, Göttingen, Germany) digital high-resolution slide scanner and reviewed using MIRAX Viewer Version 1.12 for Windows XP (Carl Zeiss MircoImaging).

The staining intensity $(0,1+, 2+, 3+)$ and the fraction of positive tumor cells were scored for each tissue spot as recently published [25]. Spots without staining and spots with a staining intensity of $1+$ in $\leq 20 \%$ of tumor cells were classified as CXCR7 negative. Spots with a staining intensity of $1+$ in $>20 \%$ tumor cells, and spots with staining intensity $\geq 2$ were classified as CXCR7 positive.

Immunohistochemical analysis of the sections was performed without knowledge of the patients' identity or clinical status.

\section{Statistical analysis}

SPSS Statistics for Windows (Version 17, SPSS Inc., Chicago, IL USA) was used for statistical analysis. Relationships between the immunostaining and clinicopathological data were calculated using Chi-square and Fisher's-Exact tests and displayed in cross tables. Group Survival curves were plotted using the KaplanMeier method and analyzed by log-rank test. All tests were two-sided and $\mathrm{p}$-values less than 0.05 were considered statistically significant.

\section{Results}

\section{Characteristics of the patients}

Tissue specimens of 299 patients aged 35 to 93 years (median 63 years) with the diagnosis of EAC (45\%) and ESCC (55\%) were included in this study. Of these, 235 men $(79 \%)$ and 64 women (21\%) were treated surgically between 1992 and 2005 in the Department of General, Visceral and Thoracic Surgery of the University Medical Center Hamburg-Eppendorf, Germany. Operation methods were transhiatal or abdominothoracic (Ivor Lewis) esophagectomy with intrathoracic or cervical anastomosis. Histopathologic findings are summarized in Table 1. Bone marrow aspirates of 190 patients were analyzed and disseminated tumor cells were identified in $34 \%$ of the patients $(n=65)$. Median follow-up time of all patients included in the survival analysis was 15 months (range 0-178 months); calculated median OS for all patients was 15.0 months. Twenty-two patients (7.4\%) died within the first 30 days after surgery.

\section{CXCR7 Expression in primary tumors, lymph nodes and distant metastases of ESC and EAC}

A total of 290 (97\%) primary EC tumor samples were interpretable in our tissue microarray (TMA) analysis. 
Table 1 Correlation between CXCR7 expression and histopathologic cell type in primary tumors (PT), lymph node (LN) and distant metastases (Met)

\begin{tabular}{|c|c|c|c|c|c|}
\hline & & \multirow[b]{2}{*}{ Total } & \multicolumn{2}{|c|}{ Histopathologic cell type } & \multirow[b]{2}{*}{$\mathrm{p}$-value } \\
\hline & & & ESCC & EAC & \\
\hline \multicolumn{6}{|c|}{ CXCR7 status } \\
\hline \multirow[t]{2}{*}{ PT } & Negative & 215 & $87(55 \%)$ & $128(98 \%)$ & \\
\hline & Positive & 75 & $72(45 \%)$ & $3(2 \%)$ & 0.000 \\
\hline \multirow[t]{2}{*}{ LN } & Negative & 96 & $33(60 \%)$ & $63(93 \%)$ & \\
\hline & Positive & 27 & $22(40 \%)$ & $5(7 \%)$ & 0.000 \\
\hline \multirow[t]{2}{*}{ Met } & Negative & 33 & $6(43 \%)$ & 27 (96\%) & \\
\hline & Positive & 9 & $8(57 \%)$ & $1(4 \%)$ & 0.000 \\
\hline
\end{tabular}

Reasons for non-informative cases $(n=9 ; 3 \%)$ included a complete lack of tissue samples or the absence of unequivocal cancer tissue in the TMA sections. Immunohistochemical staining for CXCR7 showed cytoplasmic expression of the molecule with a membranous accentuation. CXCR7 was not found in healthy esophageal mucosa cells, but only in endothelial cells $(n=10$, Figure $1 \mathrm{H}$ and 1I). The morphologic staining pattern did not differ between EAC and ESCC; both tumors showed a heterogeneous staining pattern inside the cancerous lesions (Figure 1A-G).

Only a small subset of EAC primary lesions (2\%), lymph nodes $(7 \%)$ and distant metastases (4\%) were CXCR7 positive, while nearly half of the primary ESCC expressed CXCR7 (45\%). According to this, CXCR7 expression is present significantly more often in ESCC than in EAC tumors $(\mathrm{p}<0.001)$. Of the ESCC lymph node metastases, $40 \%$ were CXCR7 positive, as well as $57 \%$ of the distant metastases (Table 1 and Figure 1A-1G).

\section{Correlation of CXCR7 expression in ESCC patients with clinical and histopathological characteristics}

Due to the fact that CXCR7 is expressed in ESCC only, no statistical analysis is presented for EAC patients. As shown in Table 1, statistical analysis of the immunohistological staining results revealed no association with the tested characteristics (age, sex, disease stage ( $\mathrm{T}, \mathrm{N}$, $M$ status), tumor grading (G), LN or Met CXCR7 status). Results of CXCR7 immunohistochemistry were correlated with the recently published data regarding CXCR4 expression [3]. In total, 95 specimens were stained for both receptors; Forty-six patients with ESCC and 49 with EACC. Only one of the CXCR4 stained EACC

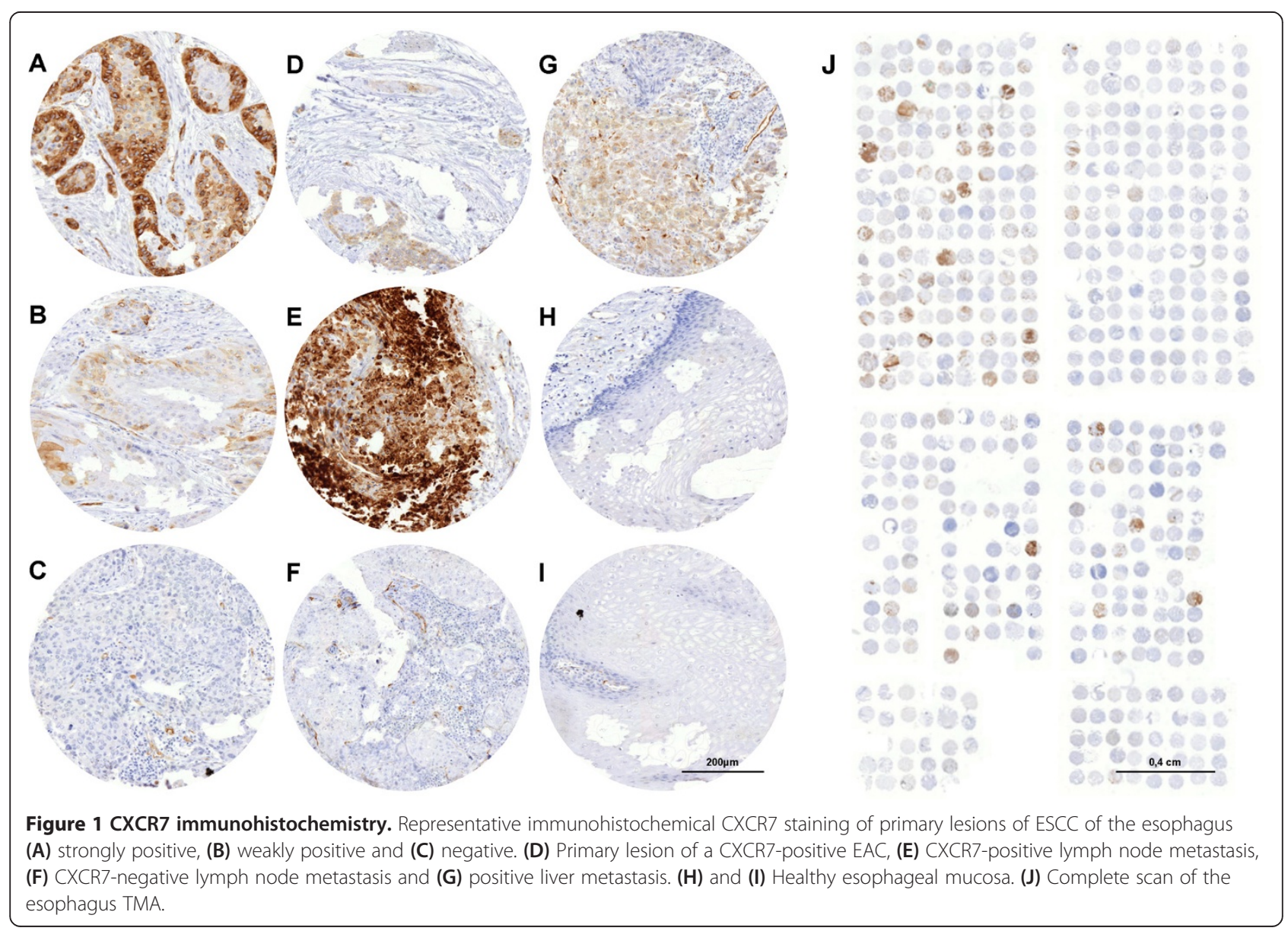


specimens was CXRC7 positive, not enough for statistical analysis. No significant correlation was seen between CXCR4 and CXCR7 expression in ESCC ( $\mathrm{p}=0.128$, Table 2).

Overall survival curves plotted by Kaplan-Meier analysis revealed no significant difference between CXCR7 negative and positive patients $(\mathrm{p}=0.469$, Figure 2$)$.

\section{Discussion}

The CXCL12/CXCR4/CXCR7 chemokine axis has been identified as an important element in tumor development, progress and metastasis [13]. In EC, several authors have investigated the expression and function of CXCL12 and CXCR4 and found pro-proliferative and metastatic

Table 2 Correlation between CXCR7 expression and clinicopathological data

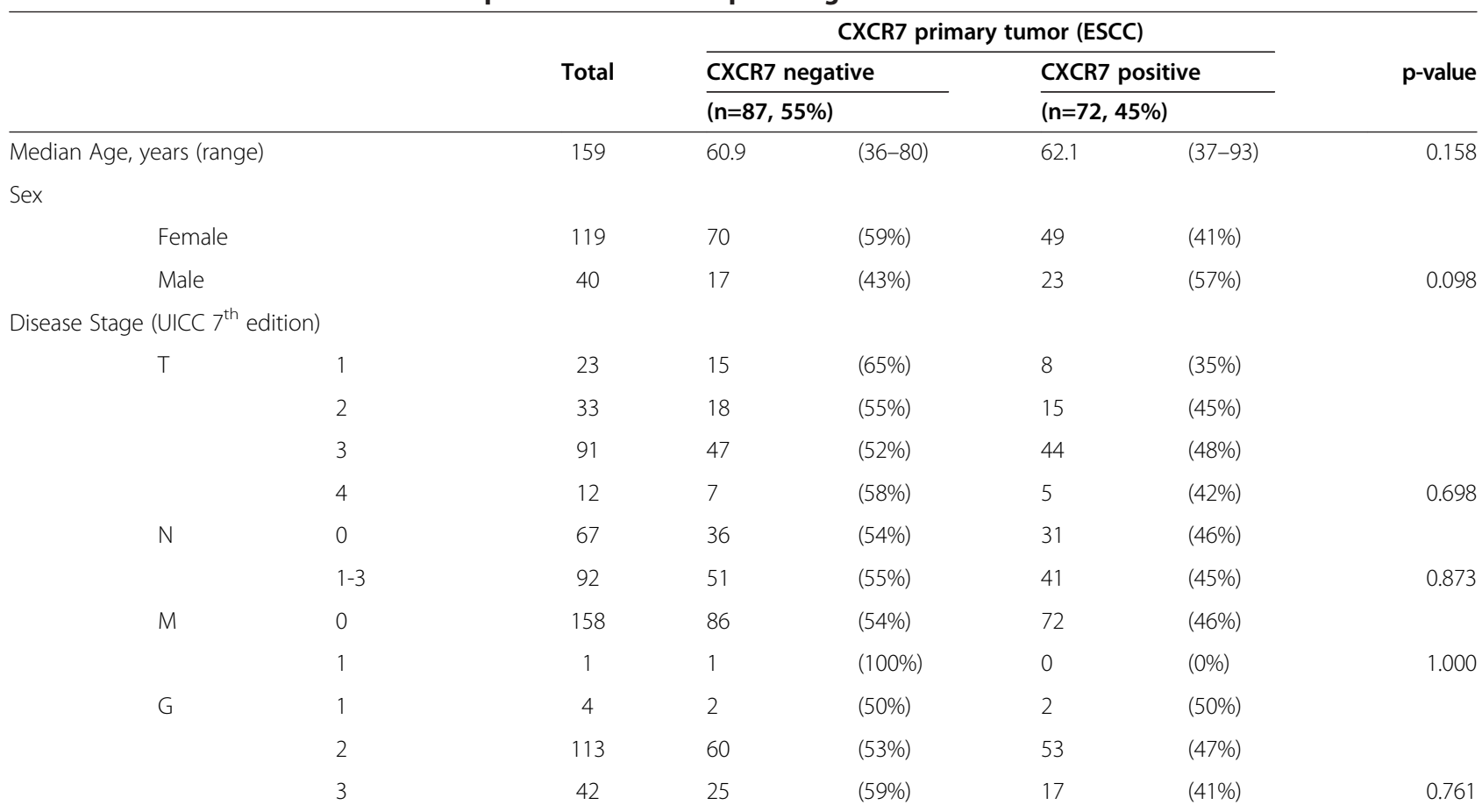

Prognostic Tumor Staging (UICC $7^{\text {th }}$ edition)

$l a^{\circ}$
$I b^{\circ}$
$\| a^{\circ}$
$\| b^{\circ}$
$\| a^{\circ}$
$\| b^{\circ}$
$\| c^{\circ}$
$I V^{\circ}$

19
16
28
13
40
20
22
1

$\begin{array}{lll}(63 \%) & 7 & (37 \%) \\ (50 \%) & 8 & (50 \%) \\ (50 \%) & 14 & (50 \%) \\ (61 \%) & 5 & (39 \%) \\ (52 \%) & 19 & (47 \%) \\ (50 \%) & 10 & (50 \%) \\ (59 \%) & 9 & (41 \%) \\ (100 \%) & 0 & (0 \%)\end{array}$

Bone marrow micrometastasis

$$
\begin{aligned}
& \text { Negative } \\
& \text { Positive }
\end{aligned}
$$

$\begin{array}{ll}61 & 34 \\ 34 & 17\end{array}$

CXCR7 status

$\begin{array}{clc}\text { LN } & \text { Negative } & 32 \\ & \text { Positive } & 20 \\ \text { Met } & \text { Negative } & 6 \\ & \text { Positive } & 8\end{array}$

$\begin{array}{lll}(53 \%) & 15 & (47 \%) \\ (45 \%) & 11 & (55 \%) \\ (67 \%) & 2 & (33 \%) \\ (50 \%) & 4 & (50 \%)\end{array}$




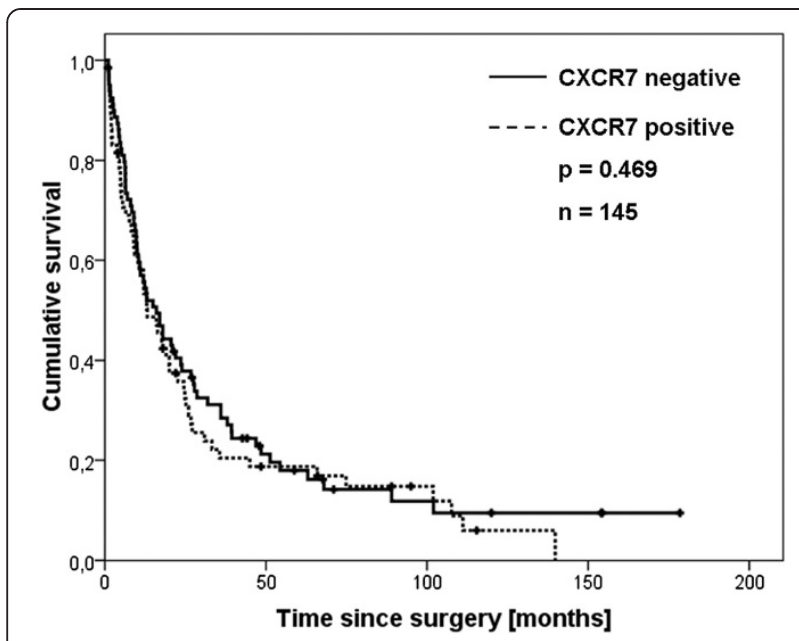

Figure 2 Kaplan-Meier overall-survival analysis for patients suffering from ESCC. Groups with and without CXCR7 expression in ESCC were compared. P-values were calculated with the log-rank test.

potential as well as a significant association with shortened OS, but no studies regarding the expression, prognostic significance or function of CXCR7 in EC have been published [3,15-20]. The current study presents the results of an immunohistochemical CXCR7 staining experiment on primary and metastatic specimens in a large cohort of EC patients.

In normal esophageal mucosa, no expression was found except in endothelial cells [26]. CXCR7 expression was rarely found in EAC (2\%), but was over-expressed in almost half of the ESCC (45\%). A similar phenomenon has been described for non-small lung cell cancer (NSCLC); CXCR7 is expressed predominantly in squamous cell carcinoma and occasionally in adenocarcinoma [27]. In contrast to this, the published studies investigating CXCR4 expression have shown a similar rate in EAC and ESCC, which might be another hint as to their divergent functional roles in EC [3,15,17]. A recently published study of our group has immunohistochemically analyzed the CXCR4 expression of esophageal cancer specimen on conventional histological slides. Ninety five of them were implemented in the CXCR7 stained TMA and analyzable. The expression analysis of both receptors revealed a statistically co-expression neither in ESCC nor in EAC. This discrepant presentation has already been shown in an expression analysis study of lung cancer specimen [28]. Based on their results, Imai and colleagues suggested that CXCL12 may differentially interact with CXCR4 or CXCR7, depending on the cellular context [28]. The molecular mechanisms of CXCR7 are poorly understood and so are its physiologic and oncologic functions in tumor development and progression, whereas a pro-proliferative role of CXCR7 for tumor cells and neovascularisation has been described $[13,26]$. CXCR7 is described as a potential, but according the results of our study not essential modulator or co-receptor for CXCR4 signaling. The infrequent expression of CXCR7 in EAC might explain why CXCR4 inhibitors have shown, due to the absence of the alternate receptor CXCR7, a significant effect in EAC in in-vitro and in-vivo experiments [16,21]. Similarly, if CXCR7 might function as an escape receptor for CXCR4, merely inhibiting CXCR4 expression might have no significant effect in ESCC.

In general, the significantly different expression rate in EAC and ESCC underlines the biologic divergence between the two types of EC tumors, which were assumed to behave in a similar fashion for a long time $[29,30]$. As a consequence, the recently published, 7th AJCC/ UICC classification has proposed different prognostic staging groups for EAC and ESCC [31].

Contrary to other entities, such as breast, prostate and lung cancer, neither was an association seen between CXCR7 expression in primary lesions and clinical and histopathological data, nor was it shown to have an impact as a prognostic marker for survival of patients with ESCC $[27,32,33]$.

\section{Conclusions}

In conclusion, the results of the study do not indicate a significant functional role for CXCR7 in EC. Nevertheless, CXCR7 is detectable in a high number of ESCC patients and since biologic functions have been reported in recent literature, it might serve as a possible target for molecular therapies.

However, the functional background for its divergent expression in EAC and ESCC and the functions and interactions in EC between CXCR7, its ligand SDF- $1 \alpha$ and the other members of the cytokine family should be further investigated.

\section{Competing interests}

The authors declare that they have no competing interests.

\section{Authors' contributions}

MT conceived the study and participated in its design and coordination, drafted the manuscript, performed the statistical analysis and collected the clinicopathological data. FG conceived the study and participated in its design and coordination and collected the clinicopathological data. HZ conceived the study and participated in its design and coordination and drafted the manuscript. MB performed the statistical analysis and participated in the design of the study and coordination and helped to draft the manuscript. KL participated in the establishment of immunohistochemistry and analyzed the arrays. KP participated in the design of the study and coordination and helped to draft the manuscript. $\mathrm{JRI}$ participated in the study design and coordination and helped to draft the manuscript. All authors read and approved the final manuscript.

\section{Acknowledgements}

We thank the patients who willingly and generously provided data and samples for research purposes, as well as Christina Koop for her excellent technical support. 


\section{Author details}

${ }^{1}$ Department of General, Visceral and Thoracic Surgery, University Medical Center Hamburg-Eppendorf, Martinistrasse 52, 20246 Hamburg, Germany. ${ }^{2}$ Department of Pathology, University Medical Center Hamburg-Eppendorf, Hamburg, Germany. ${ }^{3}$ Institute of Tumor Biology, University Medical Center Hamburg-Eppendorf, Martinistrasse 52, 20246 Hamburg, Germany.

Received: 6 May 2013 Accepted: 25 September 2013

Published: 30 September 2013

\section{References}

1. Yekebas EF, Schurr PG, Kaifi JT, Link BC, Kutup A, et al: Effectiveness of radical en-bloc-esophagectomy compared to transhiatal esophagectomy in squamous cell cancer of the esophagus is influenced by nodal micrometastases. J Surg Oncol 2006, 93:541-549.

2. Koenig AM, Prenzel KL, Bogoevski D, Yekebas EF, Bubenheim M, et al: Strong impact of micrometastatic tumor cell load in patients with esophageal carcinoma. Ann Surg Oncol 2009, 16:454-462.

3. Kaifi JT, Yekebas EF, Schurr P, Obonyo D, Wachowiak R, et al: Tumor-cell homing to lymph nodes and bone marrow and CXCR4 expression in esophageal cancer. J Natl Cancer Inst 2005, 97:1840-1847.

4. Hyngstrom JR, Posner MC: Neoadjuvant strategies for the treatment of locally advanced esophageal cancer. J Surg Oncol 2010, 101:299-304.

5. Barnett SA, Rizk NP: Randomized clinical trials in esophageal carcinoma. Surg Oncol Clin N Am 2010, 19:59-80.

6. O'Reilly S, Forastiere A: New approaches to treating oesophageal cancer. BMJ 1994, 308:1249-1250.

7. Siewert JR, Stein HJ, Feith M, Bruecher BL, Bartels $H$, et al: Histologic tumor type is an independent prognostic parameter in esophageal cancer: lessons from more than 1,000 consecutive resections at a single center in the Western world. Ann Surg 2001, 234:360-367. discussion 368-369.

8. Oshima T, Masuda M: Molecular targeted agents for gastric and gastroesophageal junction cancer. Surg Today 2012, 42:313-327.

9. Wu X, Lee VC, Chevalier E, Hwang ST: Chemokine receptors as targets for cancer therapy. Curr Pharm Des 2009, 15:742-757.

10. Balkwill F: Cancer and the chemokine network. Nat Rev Cancer 2004, 4:540-550.

11. Lazennec $G$, Richmond $A$ : Chemokines and chemokine receptors: new insights into cancer-related inflammation. Trends Mol Med 2010, 16:133-144.

12. Maksym RB, Tarnowski M, Grymula K, Tarnowska J, Wysoczynski M, et al: The role of stromal-derived factor-1-CXCR7 axis in development and cancer. Eur J Pharmacol 2009, 625:31-40.

13. Sun X, Cheng G, Hao M, Zheng J, Zhou X, et al: CXCL12 / CXCR4 / CXCR7 chemokine axis and cancer progression. Cancer Metastasis Rev 2010, 29:709-722.

14. Burns JM, Summers BC, Wang Y, Melikian A, Berahovich $R$, et al: A novel chemokine receptor for SDF-1 and I-TAC involved in cell survival, cell adhesion, and tumor development. J Exp Med 2006, 203:2201-2213.

15. Gros SJ, Kurschat N, Drenckhan A, Dohrmann T, Forberich E, et al: Involvement of CXCR4 chemokine receptor in metastastic HER2-positive esophageal cancer. PLoS One 2012, 7:e47287.

16. Gros SJ, Graeff H, Drenckhan A, Kurschat N, Blessmann M, et al: CXCR4/SDF-1alpha-mediated chemotaxis in an in vivo model of metastatic esophageal carcinoma. In Vivo 2012, 26:711-718.

17. Gockel I, Schimanski CC, Heinrich C, Wehler T, Frerichs K, et al: Expression of chemokine receptor CXCR4 in esophageal squamous cell and adenocarcinoma. BMC Cancer 2006, 6:290

18. Sasaki $K$, Natsugoe $S$, Ishigami $S$, Matsumoto $M$, Okumura $H$, et al: Expression of CXCL12 and its receptor CXCR4 correlates with lymph node metastasis in submucosal esophageal cancer. J Surg Oncol 2008, 97:433-438

19. Wang DF, Lou N, Zeng CG, Zhang X, Chen FJ: Expression of CXCL12/ CXCR4 and its correlation to prognosis in esophageal squamous cell carcinoma. Ai Zheng 2009, 28:154-158.

20. Zhang L, Ye SB, Ma G, Tang XF, Chen SP, et al: The expressions of MIF and CXCR4 protein in tumor microenvironment are adverse prognostic factors in patients with esophageal squamous cell carcinoma. J Transl Med 2013, 11:60.

21. Drenckhan A, Kurschat N, Dohrmann T, Raabe N, Koenig AM, et al: Effective inhibition of metastases and primary tumor growth with CTCE-9908 in esophageal cancer. J Surg Res 2013, 182:250-256.
22. Vashist $\mathrm{YK}$, Effenberger $\mathrm{KE}$, Vettorazzi $\mathrm{E}$, Riethdorf $\mathrm{S}$, Yekebas $\mathrm{EF}$, et al: Disseminated tumor cells in bone marrow and the natural course of resected esophageal cancer. Ann Surg 2012, 255:1105-1112.

23. Reichelt U, Duesedau P, Tsourlakis M, Quaas A, Link BC, et al: Frequent homogeneous HER-2 amplification in primary and metastatic adenocarcinoma of the esophagus. Mod Pathol 2007, 20:120-129.

24. Tachezy M, Effenberger K, Zander H, Minner S, Gebauer F, et al: ALCAM (CD166) expression and serum levels are markers for poor survival of esophageal cancer patients. Int J Cancer 2012, 131:396-405.

25. Simon R, Mirlacher M, Sauter G: Immunohistochemical analysis of tissue microarrays. Methods Mol Biol 2010, 664:113-126.

26. Sanchez-Martin L, Sanchez-Mateos P, Cabanas C: CXCR7 impact on CXCL12 biology and disease. Trends Mol Med 2013, 19:12-22.

27. Miao Z, Luker KE, Summers BC, Berahovich R, Bhojani MS, et al: CXCR7 (RDC1) promotes breast and lung tumor growth in vivo and is expressed on tumor-associated vasculature. Proc Natl Acad Sci U S A 2007, 104:15735-15740.

28. Imai H, Sunaga N, Shimizu Y, Kakegawa S, Shimizu K, et al: Clinicopathological and therapeutic significance of CXCL12 expression in lung cancer. Int J Immunopathol Pharmacol 2010, 23:153-164.

29. Torres C, Turner JR, Wang HH, Richards W, Sugarbaker D, et al: Pathologic prognostic factors in Barrett's associated adenocarcinoma: a follow-up study of 96 patients. Cancer 1999, 85:520-528

30. Theunissen PH, Borchard F, Poortvliet DC: Histopathological evaluation of oesophageal carcinoma: the significance of venous invasion. Br J Surg 1991, 78:930-932.

31. International Union Against Cancer: Oesophagus including Oesophagogastric Junction. In Classification of Malignant Tumours. 7th edition. Edited by Sobin LH, Gospodarowicz MK, Wittekind C. New York, NY: Wiley Blackwell; 2009:66-72.

32. Wang J, Shiozawa $Y$, Wang $Y$, Jung $Y$, Pienta $K$, et al: The role of CXCR7/ RDC1 as a chemokine receptor for CXCL12/SDF-1 in prostate cancer. J Biol Chem 2008, 283:4283-4294.

33. Iwakiri S, Mino N, Takahashi T, Sonobe M, Nagai S, et al: Higher expression of chemokine receptor CXCR7 is linked to early and metastatic recurrence in pathological stage I nonsmall cell lung cancer. Cancer 2009, 115:2580-2593.

doi:10.1186/1479-5876-11-238

Cite this article as: Tachezy et al:: CXCR7 expression in esophageal cancer. Journal of Translational Medicine 2013 11:238.

\section{Submit your next manuscript to BioMed Central and take full advantage of:}

- Convenient online submission

- Thorough peer review

- No space constraints or color figure charges

- Immediate publication on acceptance

- Inclusion in PubMed, CAS, Scopus and Google Scholar

- Research which is freely available for redistribution
C Biomed Central 